Electronic version of an article published as International Journal of Foundations of Computer Science, 25(07), 2014, 807-821.

http://dx.doi.org/10.1142/S0129054114400097 @ World Scientific Publishing Company http://www.worldscientific.com/

February 23, $2015 \quad$ 12:12 WSPC/INSTRUCTION FILE $\quad$ RJtrivial'̈ijfcs

International Journal of Foundations of Computer Science

(C) World Scientific Publishing Company

\title{
SYNTACTIC COMPLEXITY OF $\mathcal{R}$ - AND $\mathcal{J}$-TRIVIAL REGULAR LANGUAGES*
}

\author{
JANUSZ BRZOZOWSKI and BAIYU LI ${ }^{\dagger}$ \\ David R. Cheriton School of Computer Science, University of Waterloo \\ Waterloo, ON, Canada N2L $3 G 1$ \\ \{brzozo,b5li\}@uwaterloo.ca
}

\begin{abstract}
The syntactic complexity of a subclass of the class of regular languages is the maximal cardinality of syntactic semigroups of languages in that class, taken as a function of the state complexity $n$ of these languages. We prove that $n$ ! and $\lfloor e(n-1) !\rfloor$ are tight upper bounds for the syntactic complexity of $\mathcal{R}$ - and $\mathcal{J}$-trivial regular languages, respectively.

Keywords: finite automaton; $\mathcal{J}$-trivial; monoid; regular language; $\mathcal{R}$-trivial; semigroup; syntactic complexity.
\end{abstract}

\section{Introduction}

The state complexity of a regular language $L$ is the number of states in the minimal deterministic finite automaton (DFA) accepting $L$. An equivalent notion is quotient complexity, which is the number of distinct left quotients of $L$. The syntactic complexity of $L$ is the cardinality of the syntactic semigroup of $L$. Since the syntactic semigroup of $L$ is isomorphic to the semigroup of transformations performed by the minimal DFA of $L$, it is natural to consider the relation between syntactic complexity and state complexity. The syntactic complexity of a subclass of regular languages is the maximal syntactic complexity of languages in that class, taken as a function of the state complexity of these languages.

Here we consider the classes of languages defined using the well-known Green equivalence relations on semigroups [14]. Let $M$ be a monoid, that is, a semigroup with an identity, and let $s, t \in M$ be any two elements of $M$. The Green equivalence relations on $M$, denoted by $\mathcal{L}, \mathcal{R}, \mathcal{J}$ and $\mathcal{H}$, are defined as follows: $s \mathcal{L} t \Leftrightarrow M s=M t$, $s \mathcal{R} t \Leftrightarrow s M=t M, s \mathcal{J} t \Leftrightarrow M s M=M t M$, and $s \mathcal{H} t \Leftrightarrow s \mathcal{L} t$ and $s \mathcal{R} t$. For $\rho \in\{\mathcal{L}, \mathcal{R}, \mathcal{J}, \mathcal{H}\}, M$ is $\rho$-trivial if and only if $(s, t) \in \rho$ implies $s=t$ for all $s, t \in M$. A language is $\rho$-trivial if and only if its syntactic monoid is $\rho$-trivial. In this paper we consider only regular $\rho$-trivial languages. $\mathcal{H}$-trivial regular languages are exactly the star-free languages $[14,17]$, and $\mathcal{L}$-, $\mathcal{R}$-, and $\mathcal{J}$-trivial regular languages are all

* This work was supported by the Natural Sciences and Engineering Research Council of Canada under grant No. OGP0000871 and a Postgraduate Scholarship.

$\dagger$ Present address: Optumsoft, Inc., 275 Middlefield Rd, Suite 210, Menlo Park, CA 94025, USA 
subclasses of the class of star-free languages. The class of $\mathcal{J}$-trivial languages is the intersection of the classes of $\mathcal{R}$-trivial and $\mathcal{L}$-trivial languages.

A language $L \subseteq \Sigma^{*}$ is piecewise-testable if it is a finite boolean combination of languages of the form $\Sigma^{*} a_{1} \Sigma^{*} \cdots \Sigma^{*} a_{l} \Sigma^{*}$, where $a_{i} \in \Sigma$. Simon $[18,19]$ proved in 1972 that a language is piecewise-testable if and only if it is $\mathcal{J}$-trivial. A biautomaton is a finite automaton which can read the input word alternatively from left and right. In 2011 Klíma and Polák [10] showed that a language is piecewise-testable if and only if it is accepted by an acyclic biautomaton; here self-loops are allowed, as they are not considered cycles.

In 1979 Brzozowski and Fich [1] proved that a regular language is $\mathcal{R}$-trivial if and only if its minimal DFA is partially ordered, that is, it is acyclic as above. They also showed that $\mathcal{R}$-trivial regular languages are finite boolean combinations of languages $\Sigma_{1}^{*} a_{1} \Sigma_{2}^{*} \cdots \Sigma_{l}^{*} a_{l} \Sigma^{*}$, where $a_{i} \in \Sigma$ and $\Sigma_{i} \subseteq \Sigma \backslash\left\{a_{i}\right\}$. Recently Jirásková and Masopust proved a tight upper bound on the state complexity of reversal of $\mathcal{R}$ and $\mathcal{J}$-trivial languages $[8,9]$.

In the past, the syntactic complexity of the following subclasses of regular languages was considered: In 1970 Maslov [12] noted that $n^{n}$ was a tight upper bound on the number of transformations performed by a DFA of $n$ states. In 2003-2004, Holzer and König [7], and Krawetz, Lawrence and Shallit [11] studied unary and binary languages. In 2010 Brzozowski and Ye [5] examined ideal and closed regular languages. In 2012 Brzozowski, Li and Ye studied prefix-, suffix-, bifix-, and factor-free regular languages [4]. In 2013 Brzozowski, Li and Liu [3] considered six subclasses of star-free languages including monotonic, partially monotonic, nearly monotonic, finite/cofinite, definite, and reverse definite languages, where $L$ is definite (reverse-definite) if it can be decided whether a word $w$ belongs to $L$ by examining the suffix (prefix) of $w$ of some fixed length.

We state basic definitions and facts in Section 2. In Sections 3 and 4 we prove tight upper bounds on the syntactic complexities of $\mathcal{R}$ - and $\mathcal{J}$-trivial regular languages, respectively. Section 5 concludes the paper. A much shorter version of this work appeared in [2]; many proofs that were omitted there are given in full in the present paper.

\section{Preliminaries}

Let $Q$ be a non-empty finite set with $n$ elements, and assume without loss of generality that $Q=\{1,2, \ldots, n\}$. There is a linear order on $Q$, namely the natural order $<$ on integers. If $X$ is a non-empty subset of $Q$, then the maximal element in $X$ is denoted by $\max (X)$. A partition $\pi$ of $Q$ is a collection $\pi=\left\{X_{1}, X_{2}, \ldots, X_{m}\right\}$ of non-empty subsets of $Q$ such that $Q=X_{1} \cup X_{2} \cup \cdots \cup X_{m}$, and $X_{i} \cap X_{j}=\emptyset$ for all $1 \leqslant i<j \leqslant m$. We call each subset $X_{i}$ a block of $\pi$. For any partition $\pi$ of $Q$, let $\operatorname{Max}(\pi)=\{\max (X) \mid X \in \pi\}$. The set of all partitions of $Q$ is denoted by $\Pi_{Q}$. We define a partial order $\preceq$ on $\Pi_{Q}$ such that, for any $\pi_{1}, \pi_{2} \in \Pi_{Q}, \pi_{1} \preceq \pi_{2}$ if and

only if each block of $\pi_{1}$ is contained in some block of $\pi_{2}$. We say $\pi_{1}$ refines $\pi_{2}$ if 
$\pi_{1} \preceq \pi_{2}$. The poset $\left(\Pi_{Q}, \preceq\right)$ is a finite lattice: For any $\pi_{1}, \pi_{2} \in \Pi_{Q}$, the meet $\pi_{1} \wedge \pi_{2}$ is the $\preceq$-largest partition that refines both $\pi_{1}$ and $\pi_{2}$, and the join $\pi_{1} \vee \pi_{2}$ is the $\preceq$-smallest partition that is refined by both $\pi_{1}$ and $\pi_{2}$. From now on, we refer to the lattice $\left(\Pi_{Q}, \preceq\right)$ simply as $\Pi_{Q}$.

A transformation of a set $Q$ is a mapping of $Q$ into itself. We consider only transformations $t$ of a finite set $Q$. If $j \in Q$, then $j t$ is the image of $j$ under $t$. If $X$ is a subset of $Q$, then $X t=\{j t \mid j \in X\}$, and the restriction of $t$ to $X$, denoted by $\left.t\right|_{X}$, is a mapping from $X$ to $X t$ such that $\left.j t\right|_{X}=j t$ for all $j \in X$. The composition of transformations $t_{1}$ and $t_{2}$ of $Q$ is a transformation $t_{1} \circ t_{2}$ such that $j\left(t_{1} \circ t_{2}\right)=\left(j t_{1}\right) t_{2}$ for all $j \in Q$. We usually drop the operator "o" and write $t_{1} t_{2}$ for short. An arbitrary transformation can be written in the form

$$
t=\left(\begin{array}{ccccc}
1 & 2 & \cdots & n-1 & n \\
i_{1} & i_{2} & \cdots & i_{n-1} & i_{n}
\end{array}\right),
$$

where $i_{k}=k t, 1 \leqslant k \leqslant n$, and $i_{k} \in Q$. We also use the notation $t=\left[i_{1}, i_{2}, \ldots, i_{n}\right]$ for $t$ above. The domain $\operatorname{dom}(t)$ of $t$ is $Q$. The range $\operatorname{rng}(t)$ of $t$ is the set $\operatorname{rng}(t)=Q t$. The rank $\operatorname{rank}(t)$ of $t$ is the cardinality of $\operatorname{rng}(t)$, i.e., $\operatorname{rank}(t)=|\operatorname{rng}(t)|$. The binary relation $\omega_{t}$ on $Q \times Q$ is defined as follows: For any $i, j \in Q, i \omega_{t} j$ if and only if $i t^{k}=j t^{l}$ for some $k, l \geqslant 0$. This is an equivalence relation, and each equivalence class is called an orbit of $t$. For any $i \in Q$, the orbit of $t$ containing $i$ is denoted by $\omega_{t}(i)$. The set of all orbits of $t$ is denoted by $\Omega(t)$. Clearly, $\Omega(t)$ is a partition of $Q$.

A permutation of $Q$ is a mapping of $Q$ onto itself, so here $\operatorname{rng}(\pi)=Q$. The identity transformation 1 maps each element to itself. A transformation $t$ is a cycle of length $k$, where $k \geqslant 2$, if there exist pairwise different elements $i_{1}, \ldots, i_{k}$ such that $i_{1} t=i_{2}, i_{2} t=i_{3}, \ldots, i_{k-1} t=i_{k}$, and $i_{k} t=i_{1}$, and the remaining elements are mapped to themselves. A cycle is denoted by $\left(i_{1}, i_{2}, \ldots, i_{k}\right)$. For $i<j$, a transposition is the cycle $(i, j)$. A unitary transformation, denoted by $(j \rightarrow i)$, has $j t=i$ and $h t=h$ for all $h \neq j$. A constant transformation, denoted by $(Q \rightarrow i)$, has $j t=i$ for all $j$. A transformation $t$ is an idempotent if $t^{2}=t$. The set $\mathcal{T}_{Q}$ of all transformations of $Q$ is a finite semigroup, in fact, a monoid. We refer the reader to the book of Ganyushkin and Mazorchuk [6] for a detailed discussion of finite transformation semigroups.

For background about regular languages, we refer the reader to [20]. Let $\Sigma$ be a non-empty finite alphabet. Then $\Sigma^{*}$ is the free monoid generated by $\Sigma$, and $\Sigma^{+}$ is the free semigroup generated by $\Sigma$. A word is any element of $\Sigma^{*}$, and the empty word is $\varepsilon$. The length of a word $w \in \Sigma^{*}$ is $|w|$. A language over $\Sigma$ is any subset of $\Sigma^{*}$. The reverse of a word $w$ is denoted by $w^{R}$. For a language $L$, its reverse is $L^{R}=\left\{w \mid w^{R} \in L\right\}$. The left quotient, or simply quotient, of a language $L$ by a word $w$ is $w^{-1} L=\left\{x \in \Sigma^{*} \mid w x \in L\right\}$.

The Myhill congruence [13] $\approx_{L}$ of any language $L$ is defined as follows: $x \approx_{L} y$ if and only if $u x v \in L \Leftrightarrow u y v \in L$ for all $u, v \in \Sigma^{*}$. This congruence is also known as the syntactic congruence of $L$. The quotient set $\Sigma^{+} / \approx_{L}$ of equivalence classes of the relation $\approx_{L}$ is a semigroup called the syntactic semigroup of 
$L$, and $\Sigma^{*} / \approx_{L}$ is the syntactic monoid of $L$. The syntactic complexity $\sigma(L)$ of $L$ is the cardinality of its syntactic semigroup. A language is regular if and only if its syntactic semigroup is finite. We consider only regular languages, and so assume that all syntactic semigroups and monoids are finite.

A DFA is denoted by $\mathcal{A}=\left(Q, \Sigma, \delta, q_{1}, F\right)$, as usual. The DFA $\mathcal{A}$ accepts a word $w \in \Sigma^{*}$ if $\delta\left(q_{1}, w\right) \in F$. The language accepted by $\mathcal{A}$ is denoted by $L(\mathcal{A})$. If $q$ is a state of $\mathcal{A}$, then the language $L_{q}$ of $q$ is the language accepted by the DFA $(Q, \Sigma, \delta, q, F)$. Two states $p$ and $q$ of $\mathcal{A}$ are equivalent if $L_{p}=L_{q}$. If $L \subseteq \Sigma^{*}$ is a regular language, then its quotient $D F A$ is $\mathcal{A}=\left(Q, \Sigma, \delta, q_{1}, F\right)$, where $Q=\left\{w^{-1} L\right.$ $\left.w \in \Sigma^{*}\right\}, \delta\left(w^{-1} L, a\right)=(w a)^{-1} L, q_{1}=\varepsilon^{-1} L=L, F=\left\{w^{-1} L \mid \varepsilon \in w^{-1} L\right\}$. The quotient complexity $\kappa(L)$ of $L$ is the number of distinct quotients of $L$. The quotient DFA of $L$ is the minimal DFA accepting $L$, and so quotient complexity is the same as state complexity.

If $\mathcal{A}=\left(Q, \Sigma, \delta, q_{1}, F\right)$ is a DFA, then its transition semigroup [14], denoted by $T_{\mathcal{A}}$, consists of all transformations $t_{w}$ on $Q$ performed by non-empty words $w \in \Sigma^{+}$ such that $j t_{w}=\delta(j, w)$ for all $j \in Q$. The syntactic semigroup $T_{L}$ of a regular language $L$ is isomorphic to the transition semigroup of the quotient DFA $\mathcal{A}$ of $L$ [14], and we represent elements of $T_{L}$ by transformations in $T_{\mathcal{A}}$. Given a set $G=\left\{t_{a} \mid a \in \Sigma\right\}$ of transformations of $Q$, we can define the transition function $\delta$ of some DFA $\mathcal{A}$ such that $\delta(j, a)=j t_{a}$ for all $j \in Q$. The transition semigroup of such a DFA is the semigroup generated by $G$. When the context is clear, we write $a=t$, to mean that the transformation performed by $a \in \Sigma$ is $t$.

\section{3. $\mathcal{R}$-Trivial Regular Languages}

Given DFA $\mathcal{A}=\left(Q, \Sigma, \delta, q_{1}, F\right)$, we define the reachability relation $\rightarrow$ as follows. For all $p, q \in Q, p \rightarrow q$ if and only if $\delta(p, w)=q$ for some $w \in \Sigma^{*}$. We say that $\mathcal{A}$ is partially ordered [1] if the relation $\rightarrow$ is a partial order on $Q$.

Consider the natural order $<$ on $Q$. A transformation $t$ of $Q$ is non-decreasing if $p \leqslant p t$ for all $p \in Q$. The set $\mathcal{F}_{Q}$ of all non-decreasing transformations of $Q$ is a semigroup, since the composition of two non-decreasing transformations is again non-decreasing. It was shown in [1] that a language $L$ is $\mathcal{R}$-trivial if and only if its quotient DFA is partially ordered. Equivalently, $L$ is an $\mathcal{R}$-trivial language if and only if its syntactic semigroup contains only non-decreasing transformations.

It is known [6] that $\mathcal{F}_{Q}$ is generated by the following set

$$
\mathcal{G F}_{Q}=\{\mathbf{1}\} \cup\left\{t \in \mathcal{F}_{Q} \mid t^{2}=t \text { and } \operatorname{rank}(t)=n-1\right\} .
$$

For any transformation $t$ of $Q$, let $\operatorname{Fix}(t)=\{j \in Q \mid j t=j\}$. Then

Lemma 1. For any $t \in \mathcal{G F}_{Q}, \operatorname{rng}(t)=\operatorname{Fix}(t)$.

Proof. Pick arbitrary $t \in \mathcal{G F}_{Q}$. The claim holds trivially for $\mathbf{1}$. Assume $t \neq \mathbf{1}$. Clearly $\operatorname{Fix}(t) \subseteq \operatorname{rng}(t)$. Suppose there exists $j \in \operatorname{rng}(t)$ but $j t \neq j$. Then $h t=j$ for 
some $h \in Q$, and $h \neq j$. However, since $h t^{2}=j t \neq j=h t, t$ is not an idempotent, which is a contradiction. Therefore $\operatorname{rng}(t)=\operatorname{Fix}(t)$.

If $n=1$, then $\mathcal{F}_{Q}$ contains only the identity transformation $\mathbf{1}$, and $\mathcal{G F}_{Q}=\mathcal{F}_{Q}=$ $\{\mathbf{1}\}$. So $\left|\mathcal{G F}_{Q}\right|=\left|\mathcal{F}_{Q}\right|=1$. Let $\left(\begin{array}{c}n \\ m\end{array}\right)$ be the binomial coefficient. If $n \geqslant 2$, then we have

Lemma 2. For $n \geqslant 2,\left|\mathcal{G F}_{Q}\right|=1+\left(\begin{array}{l}n \\ 2\end{array}\right)$.

Proof. Pick $t \in \mathcal{G F}_{Q}$ such that $t \neq \mathbf{1}$. Then $\operatorname{rank}(t)=n-1$, and, by Lemma 1 , $|\operatorname{Fix}(t)|=n-1$. There is only one element $j \in Q \backslash \operatorname{Fix}(t)$, and $j<j t$. Note that $t$ is fully determined by the pair $(j, j t)$. Hence there are $\left(\begin{array}{l}n \\ 2\end{array}\right)$ different $t$. Together with the identity $\mathbf{1}$, the cardinality of $\mathcal{G F}_{Q}$ is $1+\left(\begin{array}{l}n \\ 2\end{array}\right)$.

Lemma 3. If $G \subseteq \mathcal{F}_{Q}$ and $G$ generates $\mathcal{F}_{Q}$, then $\mathcal{G F}_{Q} \subseteq G$.

Proof. Suppose there exists $t \in \mathcal{G F}_{Q}$ such that $t \notin G$. Since $G$ generates $\mathcal{F}_{Q}$, $t$ can be written as $t=g_{1} \cdots g_{k}$ for some $g_{1}, \ldots, g_{k} \in G$, where $k \geqslant 2$. Then $\operatorname{rng}\left(g_{k}\right) \supseteq \operatorname{rng}\left(g_{k-1} g_{k}\right) \supseteq \cdots \supseteq \operatorname{rng}\left(g_{1} \cdots g_{k-1} g_{k}\right)=\operatorname{rng}(t)$. Note that $\mathbf{1}$ is the only element in $\mathcal{F}_{Q}$ with range $Q$; so if $t=\mathbf{1}$, then $g_{1}=\cdots=g_{k}=\mathbf{1}$, a contradiction.

Assume $t \neq \mathbf{1}$, and $g_{i} \neq \mathbf{1}$ for all $i=1, \ldots, k$. Then $\operatorname{rank}(t)=n-1$, and $\operatorname{rng}\left(g_{1}\right)=\cdots=\operatorname{rng}\left(g_{k}\right)=\operatorname{rng}(t)$. Since each $g_{i}$ is non-decreasing, for all $p \in \operatorname{Fix}(t)$, we must have $p \in \operatorname{Fix}\left(g_{i}\right)$ as well; so $\operatorname{Fix}(t) \subseteq \operatorname{Fix}\left(g_{i}\right)$. Moreover, since $\operatorname{Fix}\left(g_{i}\right) \subseteq$ $\operatorname{rng}\left(g_{i}\right)=\operatorname{rng}(t)$ and $\operatorname{rng}(t)=\operatorname{Fix}(t)$ by Lemma $1, \operatorname{Fix}\left(g_{i}\right)=\operatorname{Fix}(t)=\operatorname{rng}(t)$. Now, let $q$ be the unique element in $Q \backslash \operatorname{Fix}(t)$. Then $q g_{1} \neq q$, and $q g_{1} \in \operatorname{Fix}\left(g_{2}\right)=\cdots=$ $\operatorname{Fix}\left(g_{k}\right)$. So $q\left(g_{1} \cdots g_{k}\right)=q g_{1}$. However, since $t=g_{1} \cdots g_{k}, q\left(g_{1} \cdots g_{k}\right)=q t$ and $q t=q g_{1}$. Hence $t=g_{1}$, and we get a contradiction again. Therefore $\mathcal{G F}_{Q} \subseteq G$.

Consequently, $\mathcal{G F}_{Q}$ is the unique minimal generator of $\mathcal{F}_{Q}$. We also have

Lemma 4. For $n \geqslant 1,\left|\mathcal{F}_{Q}\right|=n$ !.

Proof. Pick an arbitrary $t \in \mathcal{F}_{Q}$. For each $p \in Q$, since $p \leqslant p t$, pt can be chosen from $\{p, p+1, \ldots, n\}$. Hence $\left|\mathcal{F}_{Q}\right|=n !$.

Using the lemmas, we obtain our first tight upper bound:

Theorem 5. If $L \subseteq \Sigma^{*}$ is an $\mathcal{R}$-trivial regular language of quotient complexity $\kappa(L)=n \geqslant 1$, then its syntactic complexity $\sigma(L)$ satisfies $\sigma(L) \leqslant n !$, and this bound is tight if $|\Sigma| \geqslant 1$ for $n=1$ and if $|\Sigma| \geqslant 1+\left(\begin{array}{l}n \\ 2\end{array}\right)$ for $n \geqslant 2$.

Proof. Let $\mathcal{A}$ be the quotient DFA of $L$, and let $T_{L}$ be its syntactic semigroup. Then $T_{L}$ is a subset of $\mathcal{F}_{Q}$, and $\sigma(L) \leqslant n$ !.

When $n=1$, the only regular languages are $\Sigma^{*}$ or $\emptyset$, and they are both $\mathcal{R}$-trivial and meet the bound 1 . To see the bound is tight for $n \geqslant 2$, let $\mathcal{A}_{n}=(Q, \Sigma, \delta, 1,\{n\})$ 
be the DFA with alphabet $\Sigma$ of size $1+\left(\begin{array}{l}n \\ 2\end{array}\right)$ and set of states $Q=\{1, \ldots, n\}$, where each $a \in \Sigma$ defines a distinct transformation in $\mathcal{G F}_{Q}$. For each $p \in Q$, let $t_{p}=[p, n, \ldots, n]$. Since $\mathcal{G} \mathcal{F}_{Q}$ generates $\mathcal{F}_{Q}$ and $t_{p} \in \mathcal{F}_{Q}, t_{p}=e_{1} \cdots e_{k}$ for some $e_{1}, \ldots, e_{k} \in \mathcal{G F}_{Q}$, where $k$ depends on $p$. Then there exist $a_{1}, \ldots, a_{k} \in \Sigma$ such that each $a_{i}$ performs $e_{i}$ and state $p$ is reached by $w=a_{1} \cdots a_{k}$. Moreover, $n$ is the only final state of $\mathcal{A}_{n}$. Consider any non-final state $q \in Q \backslash\{n\}$. Since $t=[2,3, \ldots, n, n] \in \mathcal{F}_{Q}$, there exist $b_{1}, \ldots, b_{l} \in \Sigma$ such that the word $u=b_{1} \cdots b_{l}$ performs $t$. State $q$ can be distinguished from other non-final states by the word $u^{n-q}$. Hence $L=L\left(\mathcal{A}_{n}\right)$ has quotient complexity $\kappa(L)=n$. The syntactic monoid of $L$ is $\mathcal{F}_{Q}$, and so $\sigma(L)=n$ !. By Lemma 3 , the alphabet of $\mathcal{A}_{n}$ is minimal.

Example 6. When $n=4$, there are $4 !=24$ non-decreasing transformations of $Q=\{1,2,3,4\}$. Among them, there are 11 transformations with rank $n-1=3$. The following 6 transformations from the 11 are idempotents: $e_{1}=[1,2,4,4], e_{2}=$ $[1,3,3,4], e_{3}=[1,4,3,4], e_{4}=[2,2,3,4], e_{5}=[3,2,3,4], e_{6}=[4,2,3,4]$.

Together with the identity transformation 1 , we have the generating set $\mathcal{G F}_{Q}$ for $\mathcal{F}_{Q}$ with 7 transformations. We can then define the DFA $\mathcal{A}_{4}$ with 7 inputs as in the proof of Theorem 5; $\mathcal{A}_{4}$ is shown in Fig. 1. The quotient complexity of $L=L\left(\mathcal{A}_{4}\right)$ is 4 , and the syntactic complexity of $L$ is 24 .

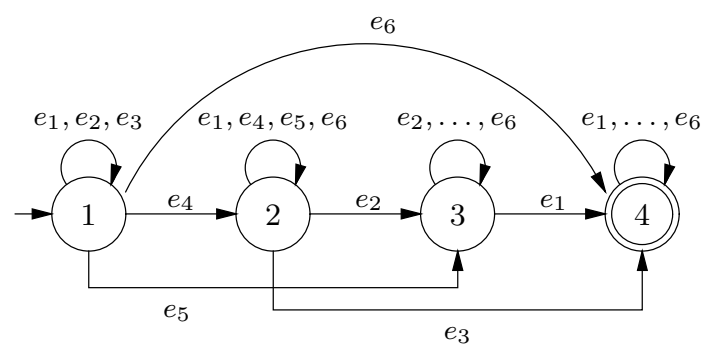

Fig. 1. DFA $\mathcal{A}_{4}$ with $\kappa\left(L\left(\mathcal{A}_{4}\right)\right)=4$ and $\sigma\left(L\left(\mathcal{A}_{4}\right)\right)=24$; the input performing the identity transformation is not shown.

\section{4. $\mathcal{J}$-Trivial Regular Languages}

For any $m \geqslant 1$, we define an equivalence relation $\leftrightarrow_{m}$ on $\Sigma^{*}$ as follows. For any $u, v \in \Sigma^{*}, u \leftrightarrow_{m} v$ if and only if for every $x \in \Sigma^{*}$ with $|x| \leqslant m, x$ is a subword of $u$ if and only if $x$ is a subword of $v$. Let $L$ be any language over $\Sigma$. Then $L$ is piecewise-testable if there exists $m \geqslant 1$ such that, for every $u, v \in \Sigma^{*}, u \leftrightarrow_{m} v$ implies that $u \in L \Leftrightarrow v \in L$. Let $\mathcal{A}=\left(Q, \Sigma, \delta, q_{1}, F\right)$ be a DFA. If $\Gamma$ is a subset of $\Sigma$, a component of $\mathcal{A}$ restricted to $\Gamma$ is a minimal subset $P$ of $Q$ such that, for all $p \in Q$ and $w \in \Gamma^{*}, \delta(p, w) \in P$ if and only if $p \in P$. A state $\mathrm{q}$ of $\mathcal{A}$ is maximal if $\delta(q, a)=q$ 
for all $a \in \Sigma$. Simon [19] proved the following characterization of piecewise-testable languages.

Theorem 7 (Simon) Let $L$ be a regular language over $\Sigma$, let $\mathcal{A}$ be its quotient $D F A$, and let $T_{L}$ be its syntactic monoid. Then the following are equivalent:

(1) $L$ is piecewise-testable.

(2) $\mathcal{A}$ is partially ordered, and for every non-empty subset $\Gamma$ of $\Sigma$, each component of $\mathcal{A}$ restricted to $\Gamma$ has exactly one maximal state.

(3) $T_{L}$ is $\mathcal{J}$-trivial.

Consequently, a regular language is piecewise-testable if and only if it is $\mathcal{J}$ trivial. The following characterization of $\mathcal{J}$-trivial monoids is due to Saito [16].

Theorem 8 (Saito) Let $S$ be a monoid of transformations of $Q$. Then the following are equivalent:

(1) $S$ is $\mathcal{J}$-trivial.

(2) $S$ is a subset of $\mathcal{F}_{Q}$ and $\Omega(t s)=\Omega(t) \vee \Omega(s)$ for all $t, s \in S$.

Let $L$ be a $\mathcal{J}$-trivial language with quotient DFA $\mathcal{A}=\left(Q, \Sigma, \delta, q_{1}, F\right)$ and syntactic monoid $T_{L}$. Since $T_{L} \subseteq \mathcal{F}_{Q}$, an upper bound on the cardinality of $\mathcal{J}$-trivial submonoids of $\mathcal{F}_{Q}$ is an upper bound on the syntactic complexity of $L$.

Lemma 9. If $t, s \in \mathcal{F}_{Q}$, then

(1) $\operatorname{Fix}(t)=\operatorname{Max}(\Omega(t))$.

(2) $\Omega(t) \preceq \Omega(s)$ implies $\operatorname{Fix}(t) \supseteq \operatorname{Fix}(s)$, where $\operatorname{Fix}(t)=\operatorname{Fix}(s)$ if and only if $\Omega(t)=\Omega(s)$.

Proof. 1. First, for each $j \in \operatorname{Max}(\Omega(t))$, since $t \in \mathcal{F}_{Q}$, we have $j t=j$, and $j \in \operatorname{Fix}(t)$. So $\operatorname{Max}(\Omega(t)) \subseteq \operatorname{Fix}(t)$. On the other hand, if there exists $j \in \operatorname{Fix}(t) \backslash$ $\operatorname{Max}(\Omega(t))$, then $j t=j$, and $j<\max \left(\omega_{t}(j)\right)$. Let $i=\max \left(\omega_{t}(j)\right)$; then $i t=i$ and, for any $k, l \geqslant 0, j t^{k}=j<i=i t^{l}$. So $i \notin \omega_{t}(j)$, which is a contradiction. Hence $\operatorname{Fix}(t)=\operatorname{Max}(\Omega(t))$.

2. Assume $\Omega(t) \preceq \Omega(s)$. By definition, we have $\operatorname{Max}(\Omega(t)) \supseteq \operatorname{Max}(\Omega(s))$. Then, by $1, \operatorname{Fix}(t) \supseteq \operatorname{Fix}(s)$. Furthermore, $\Omega(t)=\Omega(s)$ if and only if $\operatorname{Max}(\Omega(t))=$ $\operatorname{Max}(\Omega(s))$, and if and only if $\operatorname{Fix}(t)=\operatorname{Fix}(s)$.

Example 10. Consider non-decreasing transformation $t=[1,3,3,5,6,6]$, as shown in Fig. 2 (a). The orbit set $\Omega(t)$ has three blocks: $\{1\},\{2,3\}$, and $\{4,5,6\}$. Note that $\operatorname{Fix}(t)=\{1,3,6\}=\operatorname{Max}(\Omega(t))$, as expected.

Let $s=[4,3,3,6,6,6]$ be another non-decreasing transformation, as shown in Fig. 2 (b). The orbit set $\Omega(s)$ has two blocks: $\{1,4,5,6\}$ and $\{2,3\}$. Note that $\Omega(t) \prec$ $\Omega(s)$ and $\operatorname{Fix}(t) \supset \operatorname{Fix}(s)$. 
(a)

(b)

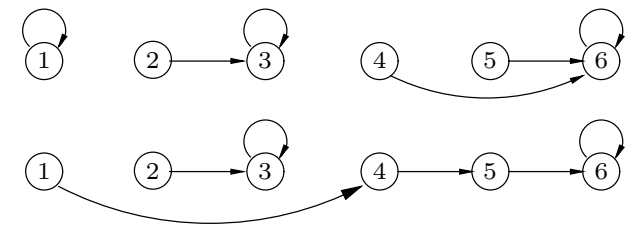

Fig. 2. Non-decreasing transformations $t=[1,3,3,5,6,6]$ and $s=[4,3,3,6,6,6]$.

Define the transformation $t_{\max }=[2,3, \ldots, n, n]$. The subscript "max" is chosen because $\Omega\left(t_{\max }\right)=\{Q\}$ is the maximal element in the lattice $\Pi_{Q}$. Clearly $t_{\max } \in \mathcal{F}_{Q}$ and $\operatorname{Fix}\left(t_{\max }\right)=\{n\}$. For any submonoid $S$ of $\mathcal{F}_{Q}$, let $S\left[t_{\max }\right]$ be the smallest monoid containing $t_{\max }$ and all elements of $S$.

Lemma 11. Let $S$ be a $\mathcal{J}$-trivial submonoid of $\mathcal{F}_{Q}$. Then

(1) $S\left[t_{\max }\right]$ is $\mathcal{J}$-trivial.

(2) Let $\mathcal{A}=(Q, \Sigma, \delta, 1,\{n\})$ be the DFA in which each $a \in \Sigma$ defines a distinct transformation in $S\left[t_{\mathrm{max}}\right]$. Then $\mathcal{A}$ is minimal.

Proof. 1. By Theorem 8 , it is sufficient to prove that for any $t \in S, \Omega(t) \vee \Omega\left(t_{\max }\right)=$ $\Omega\left(t t_{\max }\right)$ and $\Omega\left(t_{\max }\right) \vee \Omega(t)=\Omega\left(t_{\max } t\right)$. Note that $\Omega\left(t_{\max }\right)=\{Q\}$; so we have $\Omega(t) \vee \Omega\left(t_{\max }\right)=\Omega\left(t_{\max }\right) \vee \Omega(t)=\{Q\}$. On the other hand, since $S \subseteq \mathcal{F}_{Q}$ and $t_{\max } \in \mathcal{F}_{Q}$, both $t t_{\max }$ and $t_{\max } t$ are non-decreasing as well. Suppose $j \in \operatorname{Fix}\left(t t_{\max }\right)$; then $j\left(t t_{\max }\right)=(j t) t_{\max }=j$. Since $t_{\max }$ is non-decreasing, $j t \leqslant j$; and since $t$ is also non-decreasing, $j \leqslant j t$. Hence $j t=j$, and $j t_{\max }=j$, which implies that $j \in \operatorname{Fix}\left(t_{\max }\right)$ and $j=n$. Then $\operatorname{Fix}\left(t t_{\max }\right)=\{n\}$ and $\Omega\left(t t_{\max }\right)=\{Q\}$. Similarly, $\operatorname{Fix}\left(t_{\max } t\right)=\{n\}$ and $\Omega\left(t_{\max } t\right)=\{Q\}$. Therefore $S\left[t_{\max }\right]$ is also $\mathcal{J}$-trivial.

2. Suppose $a_{0} \in \Sigma$ performs the transformation $t_{\max }$. Each state $p \in Q$ can be reached from the initial state 1 by the word $u=a_{0}^{p-1}$, and $p$ accepts the word $v=a_{0}^{n-p}$, while all other states reject $v$. So $\mathcal{A}$ is minimal.

For any $\mathcal{J}$-trivial submonoid $S$ of $\mathcal{F}_{Q}$, we denote by $\mathcal{A}\left(S, t_{\max }\right)$ the DFA in Lemma 11. Then $\mathcal{A}\left(S, t_{\max }\right)$ is the quotient DFA of some $\mathcal{J}$-trivial regular language $L$. Next, we have

Lemma 12. Let $S$ be a $\mathcal{J}$-trivial submonoid of $\mathcal{F}_{Q}$. For any $t, s \in S$, if $\operatorname{Fix}(t)=$ $\operatorname{Fix}(s)$, then $\Omega(t)=\Omega(s)$.

Proof. Pick any $t, s \in S$ such that $\operatorname{Fix}(t)=\operatorname{Fix}(s)$. If $t=s$, then it is trivial that $\Omega(t)=\Omega(s)$. Assume $t \neq s$, and $\Omega(t) \neq \Omega(s)$. By Part 2 of Lemma 9, we have $\Omega(t) \nprec \Omega(s)$ and $\Omega(s) \nprec \Omega(t)$. Then there exists $i \in Q$ such that $\omega_{t}(i) \nsubseteq \omega_{s}(i)$. Let $p=\max \left(\omega_{t}(i)\right)$. We define $q \in Q$ as follows. If $\max \left(\omega_{t}(i)\right) \neq \max \left(\omega_{s}(i)\right)$, then let $q=\max \left(\omega_{s}(i)\right)$; so $q \neq p$. Otherwise $\max \left(\omega_{t}(i)\right)=\max \left(\omega_{s}(i)\right)$, and there exists $j \in \omega_{t}(i)$ such that $j \notin \omega_{s}(i)$; let $q=\max \left(\omega_{s}(j)\right)$. Now $p=\max \left(\omega_{t}(j)\right)=$ 
$\max \left(\omega_{t}(i)\right)=\max \left(\omega_{s}(i)\right)$, and since $j \notin \omega_{s}(i)$, we have $q \neq p$ as well. Note that $p, q \in \operatorname{Fix}(t)=\operatorname{Fix}(s)$ in both cases. Consider the DFA $\mathcal{A}\left(S, t_{\max }\right)$ with alphabet $\Sigma$, and suppose that $a \in \Sigma$ performs $t$ and $b \in \Sigma$ performs $s$. Let $\mathcal{B}$ be the DFA $\mathcal{A}\left(S, t_{\max }\right)$ restricted to $\{a, b\}$. Since $p \in \omega_{t}(i)$ and $q \in \omega_{s}(i), p, q$ are in the same component $P$ of $\mathcal{B}$. However, $p$ and $q$ are two distinct maximal states in $P$, which contradicts Theorem 7 . Therefore $\Omega(t)=\Omega(s)$.

Example 13. To illustrate one usage of Lemma 12, we consider two nondecreasing transformations $t=[2,2,4,4]$ and $s=[3,2,4,4]$. They have the same set of fixed points $\operatorname{Fix}(t)=\operatorname{Fix}(s)=\{2,4\}$. However, $\Omega(t)=\{\{1,2\},\{3,4\}\}$ and $\Omega(s)=\{\{2\},\{1,3,4\}\}$. By Lemma 12, $t$ and s cannot appear together in a $\mathcal{J}$-trivial monoid. Indeed, consider any minimal DFA $\mathcal{A}$ having at least two inputs a,b such that a performs $t$ and $b$ performs $s$. The DFA $\mathcal{B}$ of $\mathcal{A}$ restricted to the alphabet $\{a, b\}$ is shown in Fig. 3. There is only one component in $\mathcal{B}$, but there are two maximal states 2 and 4 . By Theorem 7, the syntactic monoid of $\mathcal{A}$ is not $\mathcal{J}$-trivial.

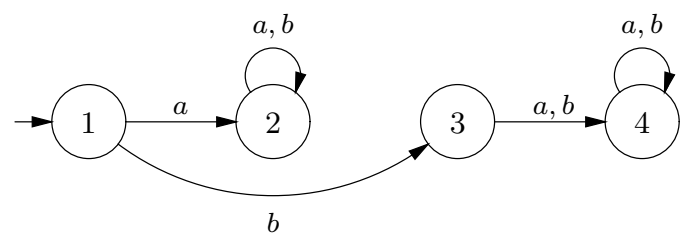

Fig. 3. DFA $\mathcal{B}$ with two inputs $a$ and $b$, where $t_{a}=[2,2,4,4]$ and $t_{b}=[3,2,4,4]$.

Let $\pi$ be any partition of $Q$. A block $X$ of $\pi$ is trivial if it contains only one element; otherwise it is non-trivial. We define the set $\mathcal{E}(\pi)=\left\{t \in \mathcal{F}_{Q} \mid \Omega(t)=\pi\right\}$. Then

Lemma 14. If $\pi$ is a partition of $Q$ with $r$ blocks, where $1 \leqslant r \leqslant n$, then $|\mathcal{E}(\pi)| \leqslant$ $(n-r)$ !. Moreover, when $r \neq n$, equality holds if and only if $\pi$ has exactly one non-trivial block.

Proof. Suppose $\pi=\left\{X_{1}, \ldots, X_{r}\right\}$, and $\left|X_{i}\right|=k_{i}$ for each $i, 1 \leqslant i \leqslant r$. Without loss of generality, we can rearrange blocks $X_{i}$ so that $k_{1} \leqslant \cdots \leqslant k_{r}$. Let $t \in \mathcal{E}(\pi)$ be any transformation. Then $t \in \mathcal{F}_{Q}$, and hence $\operatorname{Fix}(t)=\operatorname{Max}(\Omega(t))=\operatorname{Max}(\pi)$. Consider each block $X_{i}$, and suppose $X_{i}=\left\{j_{1}, \ldots, j_{k_{i}}\right\}$ with $j_{1}<\cdots<j_{k_{i}}$. Since $j_{k_{i}}=\max \left(X_{i}\right)$, we have $j_{k_{i}} \in \operatorname{Fix}(t)$ and $j_{k_{i}} t=j_{k_{i}}$. On the other hand, if $1 \leqslant l<k_{i}$, then $j_{l} \notin \operatorname{Max}(\pi)$, and since $t \in \mathcal{F}_{Q}$, we have $j_{l} t>j_{l}$; since $j_{l} t \in \omega_{t}\left(j_{l}\right)=X_{i}$, $j_{l} t \in\left\{j_{l+1}, \ldots, j_{k_{i}}\right\}$. So there are $\left(k_{i}-1\right)$ ! different $\left.t\right|_{X_{i}}$, and there are $\prod_{i=1}^{r}\left(k_{i}-1\right)$ ! different transformations $t$ in $\mathcal{E}(\pi)$.

Clearly, if $r=1$, then $k_{r}=n$ and $|\mathcal{E}(\pi)|=(n-1)$ !. Assume $r \geqslant 2$. Note that $k_{i} \geqslant 1$ for all $i, 1 \leqslant i \leqslant r$, and $\sum_{i=1}^{r} k_{i}=n$. If $k_{1}=\cdots=k_{r-1}=1$, then 
$k_{r}=n-r+1$, and $|\mathcal{E}(\pi)|=\left(k_{r}-1\right) ! \prod_{i=1}^{r-1} 0 !=(n-r)$ !. Otherwise, let $h$ be the smallest index such that $k_{h}>1$. For all $i, h \leqslant i \leqslant r-1$, since $k_{i} \leqslant k_{r}$, we have $\left(k_{i}-1\right) !<\left(k_{i}-1\right)^{k_{i}-1} \leqslant\left(k_{r}-1\right)^{k_{i}-1}$. Then

$$
\begin{aligned}
|\mathcal{E}(\pi)| & =\left(k_{r}-1\right) ! \prod_{i=1}^{h-1} 0 ! \prod_{i=h}^{r-1}\left(k_{i}-1\right) !<\left(k_{r}-1\right) ! \prod_{i=h}^{r-1}\left(k_{r}-1\right)^{k_{i}-1} \\
& =\left(k_{r}-1\right) ! \cdot\left(k_{r}-1\right)^{\sum_{i=h}^{r-1}\left(k_{i}-1\right)} \\
& <\left(k_{r}-1\right) ! \cdot k_{r}\left(k_{r}+1\right) \cdots\left(k_{r}-1+\sum_{i=h}^{r-1}\left(k_{i}-1\right)\right) \\
& =\left(k_{r}-1\right) ! \cdot k_{r}\left(k_{r}+1\right) \cdots(n-r)=(n-r) !
\end{aligned}
$$

Therefore the lemma holds.

Example 15. Suppose $n=10, r=3$, and consider the partition $\pi=\left\{X_{1}, X_{2}, X_{3}\right\}$, where $X_{1}=\{1,2,5\}, X_{2}=\{3,7\}$, and $X_{3}=\{4,6,8,9,10\}$. Then $k_{1}=\left|X_{1}\right|=3$, $k_{2}=\left|X_{2}\right|=2$, and $k_{3}=\left|X_{3}\right|=5$. Let $t \in \mathcal{E}(\pi)$ be an arbitrary transformation; then $\operatorname{Fix}(t)=\{5,7,10\}$. For any $j \in X_{1}$, if $j=1$, then $j$ t could be 2 or 5 ; otherwise $j=2$ or 5 , and $j t$ must be 5 . So there are $\left(k_{1}-1\right) !=2$ ! different $\left.t\right|_{X_{1}}$. Similarly, there are $\left(k_{2}-1\right) !=1$ ! different $\left.t\right|_{X_{2}}$ and $\left(k_{3}-1\right) !=4$ ! different $\left.t\right|_{X_{3}}$. So $|\mathcal{E}(\pi)|=2 ! 1 ! 4 !=48$.

Consider another partition $\pi^{\prime}=\left\{X_{1}^{\prime}, X_{2}^{\prime}, X_{3}^{\prime}\right\}$ with three blocks, where $X_{1}^{\prime}=\{5\}$, $X_{2}^{\prime}=\{7\}$, and $X_{3}^{\prime}=\{1,2,3,4,6,8,9,10\}$. Now $k_{1}=\left|X_{1}^{\prime}\right|=1, k_{2}=\left|X_{2}^{\prime}\right|=1$, and $k_{3}=\left|X_{3}^{\prime}\right|=8$. We have $\operatorname{Max}\left(\pi^{\prime}\right)=\operatorname{Max}(\pi)=\{5,7,10\}$. Then, for any $t \in \mathcal{E}\left(\pi^{\prime}\right)$, $\operatorname{Fix}(t)=\{5,7,10\}$ as well. Since $k_{1}=k_{2}=1$, both $\left.t\right|_{X_{1}}$ and $\left.t\right|_{X_{2}}$ are unique. There are $\left(k_{3}-1\right) !=7$ ! different $\left.t\right|_{X_{3}}$. Together we have $\left|\mathcal{E}\left(\pi^{\prime}\right)\right|=1 ! 1 ! 7 !=(10-3) !=5040$, which is the upper bound in Lemma 14 for $n=10$ and $r=3$.

Note that, for any $t \in \mathcal{F}_{Q}$, we have $n \in \operatorname{Fix}(t)$. Let $\mathcal{P}_{n}(Q)$ be the set of all subsets $Z$ of $Q$ such that $n \in Z$. Then we obtain the following upper bound.

Proposition 16. For $n \geqslant 1$, if $S$ is a $\mathcal{J}$-trivial submonoid of $\mathcal{F}_{Q}$, then

$$
|S| \leqslant \sum_{r=1}^{n}\left(\begin{array}{l}
n-1 \\
r-1
\end{array}\right)(n-r) !=\lfloor e(n-1) !\rfloor .
$$

Proof. Assume $S$ is a $\mathcal{J}$-trivial submonoid of $\mathcal{F}_{Q}$. For any $Z \in \mathcal{P}_{n}(Q)$, let $S_{Z}=$ $\{t \in S \mid \operatorname{Fix}(t)=Z\}$. Then $S=\bigcup_{Z \in \mathcal{P}_{n}(Q)} S_{Z}$, and for any $Z_{1}, Z_{2} \in \mathcal{P}_{n}(Q)$ with $Z_{1} \neq Z_{2}, S_{Z_{1}} \cap S_{Z_{2}}=\emptyset$.

Pick any $Z \in \mathcal{P}_{n}(Q)$. By Lemma 12 , for any $t, s \in S_{Z}$, since $\operatorname{Fix}(t)=\operatorname{Fix}(s)=Z$, we have $\Omega(t)=\Omega(s)=\pi$ for some partition $\pi \in \Pi_{Q}$. Then $S_{Z} \subseteq \mathcal{E}(\pi)$. Suppose $r=|Z|$. By Lemma $14,\left|S_{Z}\right| \leqslant|\mathcal{E}(\pi)| \leqslant(n-r)$ !. Since $n \in Z, 1 \leqslant r \leqslant n$; and since there are $\left(\begin{array}{l}n-1 \\ r-1\end{array}\right)$ different $Z \in \mathcal{P}_{n}(Q)$, we have

$$
|S|=\sum_{Z \in \mathcal{P}_{n}(Q)}\left|S_{Z}\right| \leqslant \sum_{r=1}^{n}\left(\begin{array}{l}
n-1 \\
r-1
\end{array}\right)(n-r) !=\sum_{r=1}^{n} \frac{(n-1) !}{(r-1) !}=\lfloor e(n-1) !\rfloor .
$$


The last equality is a well-known identity in combinatorics [15].

The above upper bound is met by the following monoid $\mathcal{S}_{n}$. For any $Z \in \mathcal{P}_{n}(Q)$, suppose $Z=\left\{j_{1}, \ldots, j_{r}, n\right\}$ such that $j_{1}<\cdots<j_{r}<n$ for some $r \geqslant 0$; then we define partition $\pi_{Z}=\{Q\}$ if $Z=\{n\}$, and $\pi_{Z}=\left\{\left\{j_{1}\right\}, \ldots,\left\{j_{r}\right\}, Q \backslash\left\{j_{1}, \ldots, j_{r}\right\}\right\}$ otherwise. Let

$$
\mathcal{S}_{n}=\bigcup_{Z \in \mathcal{P}_{n}(Q)} \mathcal{E}\left(\pi_{Z}\right)
$$

Example 17. Suppose $n=4$; then $\left|\mathcal{P}_{4}(Q)\right|=2^{3}=8$. First consider $Z=$ $\{1,3,4\} \in \mathcal{P}_{4}(Q)$. By definition, $\pi_{Z}=\{\{1\},\{3\},\{2,4\}\}$. There is only one transformation $t_{1}=[1,4,3,4]$ in $\mathcal{E}\left(\pi_{Z}\right)$. If $Z^{\prime}=\{3,4\}$, then $\pi_{Z^{\prime}}=\{\{3\},\{1,2,4\}$. There are two transformations $t_{2}=[2,4,3,4]$ and $t_{3}=[4,4,3,4]$ in $\mathcal{E}\left(\pi_{Z^{\prime}}\right)$. Table 1 summarizes the number of transformations in $\mathcal{E}\left(\pi_{Z}\right)$ for each $Z \in \mathcal{P}_{4}(Q)$. Note that the set $\mathcal{S}_{4}$ contains 16 transformations in total.

Table 1. Number of transformations in $\mathcal{E}\left(\pi_{Z}\right)$ for each $Z \in \mathcal{P}_{4}(Q)$.

\begin{tabular}{|c|c|c|}
\hline$Z$ & Blocks of $\pi_{Z}$ & $\left|\mathcal{E}\left(\pi_{Z}\right)\right|$ \\
\hline \hline$\{1,2,3,4\}$ & $\{1\},\{2\},\{3\},\{4\}$ & 1 \\
\hline$\{1,2,4\}$ & $\{1\},\{2\},\{3,4\}$ & 1 \\
\hline$\{1,3,4\}$ & $\{1\},\{3\},\{2,4\}$ & 1 \\
\hline$\{2,3,4\}$ & $\{2\},\{3\},\{1,4\}$ & 1 \\
\hline$\{1,4\}$ & $\{1\},\{2,3,4\}$ & 2 \\
\hline$\{2,4\}$ & $\{2\},\{1,3,4\}$ & 2 \\
\hline$\{3,4\}$ & $\{3\},\{1,2,4\}$ & 2 \\
\hline$\{4\}$ & $\{1,2,3,4\}$ & 6 \\
\hline
\end{tabular}

Proposition 18. For $n \geqslant 1$, the set $\mathcal{S}_{n}$ is a $\mathcal{J}$-trivial submonoid of $\mathcal{F}_{Q}$ with cardinality

$$
\left|\mathcal{S}_{n}\right|=\sum_{r=1}^{n}\left(\begin{array}{l}
n-1 \\
r-1
\end{array}\right)(n-r) !=\lfloor e(n-1) !\rfloor
$$

Proof. First we prove the following claim:

Claim: For any $t, s \in \mathcal{S}_{n}, \Omega(t s)=\pi_{Z}$ for some $Z \in \mathcal{P}_{n}(Q)$.

Let $t \in \mathcal{E}\left(\pi_{Z_{1}}\right)$ and $s \in \mathcal{E}\left(\pi_{Z_{2}}\right)$ for some $Z_{1}, Z_{2} \in \mathcal{P}_{n}(Q)$. Suppose $\Omega(t s) \neq \pi_{Z}$ for any $Z \in \mathcal{P}_{n}(Q)$. Then there exists a block $X_{0} \in \Omega(t s)$ such that $n \notin X_{0}$ and $\left|X_{0}\right| \geqslant 2$. Let $h=\max \left(X_{0}\right)$; then $h(t s)=h$, and since both $t$ and $s$ are nondecreasing, $h t=h$ and $h s=h$. Since $h \neq n$, both $\omega_{t}(h)$ and $\omega_{s}(h)$ are trivial blocks. Now let $j \in X_{0}$ such that $j(t s)=h$ and $j \neq h$. If $j t \neq h$, then $j t \in \omega_{s}(h)$ 
and $\omega_{s}(h)$ is a non-trivial block, a contradiction. Otherwise $j t=h$, then $\omega_{t}(h)$ is a non-trivial block, a contradiction again. So the claim holds.

By the claim, for any $t, s \in \mathcal{S}_{n}$, since $\Omega(t s)=\pi_{Z}$ for some $Z \in \mathcal{P}_{n}(Q)$, ts $\in$ $\mathcal{E}\left(\pi_{Z}\right) \subseteq \mathcal{S}_{n}$. Hence $\mathcal{S}_{n}$ is a submonoid of $\mathcal{F}_{Q}$.

Next we show that $\mathcal{S}_{n}$ is $\mathcal{J}$-trivial. Pick any $t, s \in \mathcal{S}_{n}$, and suppose $t \in \mathcal{E}\left(\pi_{Z_{1}}\right)$ and $s \in \mathcal{E}\left(\pi_{Z_{2}}\right)$ for some $Z_{1}, Z_{2} \in \mathcal{P}_{n}(Q)$. Suppose $\operatorname{Max}\left(Z_{1}\right) \cap \operatorname{Max}\left(Z_{2}\right)=$ $\left\{j_{1}, \ldots, j_{r}, n\right\}$, for some $r \geqslant 0$. Then we have $Z_{1} \vee Z_{2}=\left\{\left\{j_{1}\right\}, \ldots,\left\{j_{r}\right\}, X\right\}$, where $X=Q \backslash\left\{j_{1}, \ldots, j_{r}\right\}$ and $n \in X$. On the other hand, by the claim, $\Omega(t s)=\left\{\left\{p_{1}\right\}, \ldots,\left\{p_{k}\right\}, Y\right\}$ for some $p_{1}, \ldots, p_{k} \in Q$, where $Y=Q \backslash\left\{p_{1}, \ldots, p_{k}\right\}$ and $n \in Y$. Note that, since $\mathcal{S}_{n} \subseteq \mathcal{F}_{Q}, \operatorname{Max}(\Omega(t s))=\operatorname{Fix}(t s)=\operatorname{Fix}(t) \cap$ $\operatorname{Fix}(s)=\operatorname{Max}\left(Z_{1}\right) \cap \operatorname{Max}\left(Z_{2}\right)$. Then $r=k$ and $\left\{j_{1}, \ldots, j_{r}\right\}=\left\{p_{1}, \ldots, p_{k}\right\}$. Hence $\Omega(t) \vee \Omega(s)=Z_{1} \vee Z_{2}=\Omega(t s)$. By Theorem $8, \mathcal{S}_{n}$ is $\mathcal{J}$-trivial.

For any $Z \in \mathcal{P}_{n}(Q)$ with $|Z|=r$, where $1 \leqslant r \leqslant n$, we have $\pi_{Z}=\left\{X_{1}, \ldots, X_{r}\right\}$ with $k_{i}=\left|X_{i}\right|=1$ for $1 \leqslant i<r$, and $k_{r}=\left|X_{r}\right|$. By Lemma $14,\left|\mathcal{E}\left(\pi_{Z}\right)\right|=(n-r)$ !. Moreover, if $Z_{1} \neq Z_{2}$, then $\mathcal{E}\left(\pi_{Z_{1}}\right) \cap \mathcal{E}\left(\pi_{Z_{2}}\right)=\emptyset$. Since $n \in Z$ is fixed, there are $\left(\begin{array}{l}n-1 \\ r-1\end{array}\right)$ different $Z$. Therefore $\left|\mathcal{S}_{n}\right|=\sum_{r=1}^{n}\left(\begin{array}{l}n-1 \\ r-1\end{array}\right)(n-r) !=\lfloor e(n-1)$ ! $\rfloor$.

We now define a generating set of the monoid $\mathcal{S}_{n}$. Suppose $n \geqslant 1$. For any $Z \in \mathcal{P}_{n}(Q)$, if $Z=Q$, then let $t_{Z}=\mathbf{1}$. Otherwise, let $h_{Z}=\max (Q \backslash Z)$, and let $t_{Z}$ be a transformation of $Q$ defined by: For all $j \in Q$,

$$
j t_{Z} \stackrel{\text { def }}{=} \begin{cases}j & \text { if } j \in Z, \\ n & \text { if } j=h_{Z}, \\ h_{Z} & \text { otherwise. }\end{cases}
$$

Let $\mathcal{G S}_{n}=\left\{t_{Z} \mid Z \in \mathcal{P}_{n}(Q)\right\}$.

Proposition 19. For $n \geqslant 1$, the monoid $\mathcal{S}_{n}$ can be generated by the set $\mathcal{G S}_{n}$ of $2^{n-1}$ transformations of $Q$.

Proof. First, for any $t_{Z} \in \mathcal{G} \mathcal{S}_{n}$, where $Z \in \mathcal{P}_{n}(Q)$, we have $\Omega\left(t_{Z}\right)=\pi_{Z}$; hence $t_{Z} \in$ $\mathcal{E}\left(\pi_{Z}\right) \subseteq \mathcal{S}_{n}$. So $\mathcal{G S}_{n} \subseteq \mathcal{S}_{n}$ and $\left\langle\mathcal{G} \mathcal{S}_{n}\right\rangle \subseteq \mathcal{S}_{n}$, where $\left\langle\mathcal{G} \mathcal{S}_{n}\right\rangle$ denotes the semigroup generated by $\mathcal{G S}_{n}$.

Fix arbitrary $Z \in \mathcal{P}_{n}(Q)$, and suppose $U=Q \backslash Z$. If $U=\emptyset$, then $\pi_{Z}=$ $\{\{1\}, \ldots,\{n\}\}$ and $\mathcal{E}\left(\pi_{Z}\right)=\{\mathbf{1}\} \subseteq\left\langle\mathcal{G S}_{n}\right\rangle$. Assume $U \neq \emptyset$ in the following. Let $Y$ be the only non-trivial block in $\pi_{Z}$. Note that $Y=U \cup\{n\}$ and $h_{Z}=\max (U)$. For any $t \in \mathcal{E}\left(\pi_{Z}\right)$, since $\operatorname{Fix}(t)=Z$ and $h_{Z} \notin Z, h_{Z} t>h_{Z}$; and since $Y$ is an orbit of $t, h_{Z} t=n$. We prove by induction on $|U|=|Q \backslash Z|$ that $\mathcal{E}\left(\pi_{Z}\right) \subseteq\left\langle\mathcal{G} \mathcal{S}_{n}\right\rangle$.

(1) If $U=\left\{h_{Z}\right\}$, then $Y=\left\{h_{Z}, n\right\}$. So $t=\left(h_{Z} \rightarrow n\right)=t_{Z} \subseteq\left\langle\mathcal{G} \mathcal{S}_{n}\right\rangle$.

(2) Otherwise $U=\left\{h_{1}, \ldots, h_{l}, h_{Z}\right\}$ for some $h_{1}<\cdots<h_{l}<h_{Z}<n$ and $l \geqslant 1$. Assume that, for any $Z^{\prime} \in \mathcal{P}_{n}(Q)$ with $\left|Q \backslash Z^{\prime}\right| \leqslant l$, we have $\mathcal{E}\left(\pi_{Z^{\prime}}\right) \subseteq\left\langle\mathcal{G S}_{n}\right\rangle$. Then $Y=\left\{h_{1}, \ldots, h_{l}, h_{Z}, n\right\}$, and $t_{Z}=\left(h_{Z} \rightarrow n\right)\left(h_{l} \rightarrow h_{Z}\right) \cdots\left(h_{1} \rightarrow\right.$ $\left.h_{Z}\right)$. For any $t \in \mathcal{E}\left(\pi_{Z}\right)$, since $Y$ is an orbit of $t$ and $Q \backslash Y \subseteq \operatorname{Fix}(t), t$ 
must have the form $t=\left(h_{Z} \rightarrow n\right)\left(h_{l} \rightarrow j_{l}\right) \cdots\left(h_{1} \rightarrow j_{1}\right)$, where $j_{i} \in$ $\left\{h_{i+1}, \ldots, h_{l}, h_{Z}, n\right\}$ for $i=1, \ldots, l$. Let $\left\{h_{1}, \ldots, h_{l}\right\}=V \cup W$ such that $h_{i} \in V$ if and only if $j_{i}=h_{i} t=h_{Z}$. Suppose $V=\left\{h_{p_{1}}, \ldots, h_{p_{k}}\right\}$ and $W=$ $\left\{h_{q_{1}}, \ldots, h_{q_{m}}\right\}$, where $h_{p_{1}}<\cdots<h_{p_{k}}, h_{q_{1}}<\cdots<h_{q_{m}}, 0 \leqslant k, m \leqslant l$ and $l=k+m$. Let $t_{1}=\left(h_{Z} \rightarrow n\right), t_{2}=\left(h_{Z} \rightarrow n\right)\left(h_{p_{1}} \rightarrow h_{Z}\right) \cdots\left(h_{p_{k}} \rightarrow h_{Z}\right)$, and $t_{3}=\left(h_{p_{1}} \rightarrow n\right) \cdots\left(h_{p_{k}} \rightarrow n\right)\left(h_{q_{m}} \rightarrow j_{q_{m}}\right) \cdots\left(h_{q_{1}} \rightarrow j_{q_{1}}\right)$. Note that $t_{1}=t_{Z^{\prime}}$ for $Z^{\prime}=Q \backslash\left\{h_{Z}\right\}$, and $t_{2}=t_{Z^{\prime \prime}}$ for $Z^{\prime \prime}=Q \backslash\left\{h_{p_{1}}, \ldots, h_{p_{k}}, h_{Z}\right\}$. Also note that $\operatorname{Fix}\left(t_{3}\right)=\operatorname{Fix}(t) \cup\left\{h_{Z}\right\}$, and since $j_{q_{i}}=h_{q_{i}} t \in U \backslash\left\{h_{Z}\right\}$ for all $h_{q_{i}} \in W$, we have $t_{3} \in \mathcal{E}\left(\pi_{Z^{\prime \prime \prime}}\right)$ for $Z^{\prime \prime \prime}=Z \cup\left\{h_{Z}\right\}$. By assumption, $t_{3} \in\left\langle\mathcal{G} \mathcal{S}_{n}\right\rangle$. Now

$$
\begin{aligned}
t_{1} t_{2} t_{3}= & \left(h_{Z} \rightarrow n\right) \circ\left(h_{Z} \rightarrow n\right)\left(h_{p_{1}} \rightarrow h_{Z}\right) \cdots\left(h_{p_{k}} \rightarrow h_{Z}\right) \\
& \circ\left(h_{p_{1}} \rightarrow n\right) \cdots\left(h_{p_{k}} \rightarrow n\right)\left(h_{q_{m}} \rightarrow j_{q_{m}}\right) \cdots\left(h_{q_{1}} \rightarrow j_{q_{1}}\right) \\
= & \left(h_{Z} \rightarrow n\right)\left(h_{p_{1}} \rightarrow h_{Z}\right) \cdots\left(h_{p_{k}} \rightarrow h_{Z}\right)\left(h_{q_{m}} \rightarrow j_{q_{m}}\right) \cdots\left(h_{q_{1}} \rightarrow j_{q_{1}}\right) \\
= & t .
\end{aligned}
$$

Thus $t \in\left\langle\mathcal{G} \mathcal{S}_{n}\right\rangle$ and $\mathcal{E}\left(\pi_{Z}\right) \subseteq\left\langle\mathcal{G S}_{n}\right\rangle$.

By induction, $\mathcal{S}_{n}=\bigcup_{Z \in \mathcal{P}_{n}(Q)} \mathcal{E}\left(\pi_{Z}\right) \subseteq\left\langle\mathcal{G S}_{n}\right\rangle$. Therefore $\mathcal{S}_{n}=\left\langle\mathcal{G} \mathcal{S}_{n}\right\rangle$. Since there are $2^{n-1}$ different $Z \in \mathcal{P}_{n}(Q)$, there are $2^{n-1}$ transformations in $\mathcal{G} \mathcal{S}_{n}$.

Example 20. Suppose $n=5$. Consider $Z=\{3,5\} \in \mathcal{P}_{5}(Q)$, and $t=[2,4,3,5,5] \in$ $\mathcal{E}\left(\pi_{Z}\right)$. The transition graph of $t$ is shown in Fig. 4 (a). As in Proposition 19, we have $U=\{1,2,4\}$ and $h_{Z}=4$. To show that $t \in\left\langle\mathcal{G S}_{5}\right\rangle$, we find $V=\{2\}$ and $W=$ $\{1\}$. Then let $t_{1}=(4 \rightarrow 5), t_{2}=(4 \rightarrow 5)(2 \rightarrow 4)$, and $t_{3}=(2 \rightarrow 5)(1 \rightarrow 2)$. We assume that $t_{3} \in\left\langle\mathcal{G S}_{5}\right\rangle$; in fact, $t_{3}=t_{Z^{\prime \prime \prime}}$ for $Z^{\prime \prime \prime}=\{3,4,5\}$ in this example. The transition graphs of $t_{1}, t_{2}$, and $t_{3}$ are shown in Fig. $4(b),(c)$, and (d), respectively. One can verify that $t=t_{1} t_{2} t_{3}$, and hence $t \in\left\langle\mathcal{G S}_{5}\right\rangle$.

(a)
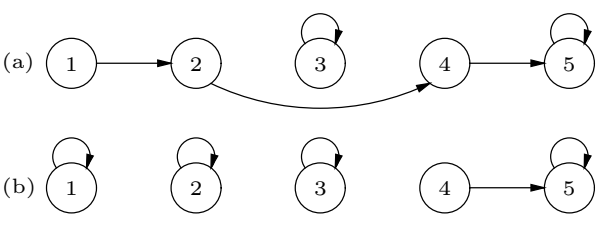

(c) 1
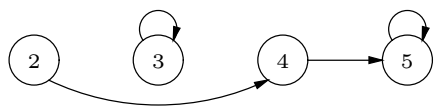

(d)

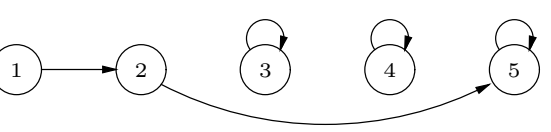

Fig. 4. Transition graphs of $t=[2,4,3,5,5], t^{\prime}=[1,4,3,5,5]$, and $t_{Z^{\prime \prime}}=[2,5,3,4,5]$. 
Now, by Propositions 16, 18, and 19, we have

Theorem 21. Let $L \subseteq \Sigma^{*}$ be a $\mathcal{J}$-trivial regular language with quotient complexity $n \geqslant 1$. Then its syntactic complexity $\sigma(L)$ satisfies $\sigma(L) \leqslant\lfloor e(n-1) !\rfloor$, and this bound is tight if $|\Sigma| \geqslant 2^{n-1}$.

It was shown by Saito [16] that, if $S$ is a $\mathcal{J}$-trivial submonoid of $\mathcal{F}_{Q}$, then $\Omega(S)=\{\Omega(t) \mid t \in S\} \subseteq \Pi_{Q}$ forms a $\vee$-semilattice, called a $\mathcal{J}$ - $\vee$-semilattice, such that $\operatorname{Max}(\Omega(t) \vee \Omega(s))=\operatorname{Fix}(t) \cap \operatorname{Fix}(s)$. Let $\mathcal{P}_{\vee}\left(\Pi_{Q}\right)$ be the set of all $\mathcal{J}$ $\vee$-semilattices that are subsets of $\Pi_{Q}$. A maximal $\mathcal{J}$-trivial submonoid $S$ of $\mathcal{F}_{Q}$ corresponds to a maximal element $P$ in $\mathcal{P}_{\vee}\left(\Pi_{Q}\right)$, with respect to set inclusion, such that $S=\bigcup_{\pi \in P} \mathcal{E}(\pi) . P \in \mathcal{P}_{\vee}\left(\Pi_{Q}\right)$ is called full if $\{\operatorname{Max}(\pi) \mid \pi \in P\}=$ $\mathcal{P}_{n}(Q)$, which is an maximal element in $\mathcal{P}_{\vee}\left(\Pi_{Q}\right)$ with respect to set inclusion. The monoid $\mathcal{S}_{n}$ then corresponds to a full $\mathcal{J}$ - $\vee$-semilattice, and hence it is maximal. Saito described all maximal $\mathcal{J}$-trivial submonoid of $\mathcal{F}_{Q}$ and those corresponding to full $\mathcal{J}$ - $\vee$-semilattices. However, here we consider the $\mathcal{J}$-trivial submonoid of $\mathcal{F}_{Q}$ with maximum cardinality.

\section{Conclusion}

We proved that $n$ ! and $\lfloor e(n-1) !\rfloor$ are the tight upper bounds on the syntactic complexities of $\mathcal{R}$ - and $\mathcal{J}$-trivial languages with $n$ quotients, respectively. For $n \geqslant 2$, the upper bound for $\mathcal{R}$-trivial languages can be met using $1+\left(\begin{array}{c}n \\ 2\end{array}\right)$ letters, and the upper bound for $\mathcal{J}$-trivial languages, using $2^{n-1}$ letters. It remains open whether the upper bound for $\mathcal{J}$-trivial languages can be met with fewer than $2^{n-1}$ letters. The syntactic complexity of $\mathcal{L}$-trivial languages is also open.

\section{References}

[1] J. Brzozowski and F. E. Fich, Languages of $\mathcal{R}$-trivial monoids, J. Comput. System Sci. 20(1) (1980) 32-49.

[2] J. Brzozowski and B. Li, Syntactic complexity of $\mathcal{R}$ - and $\mathcal{J}$-trivial regular languages, DCFS 2013, eds. H. Jurgensen and R. Reis LNCS 8031, (Springer, 2013), pp. 160171.

[3] J. Brzozowski, B. Li and D. Liu, Syntactic complexities of six classes of star-free languages, J. Autom. Lang. Comb. (2013) To appear.

[4] J. Brzozowski, B. Li and Y. Ye, Syntactic complexity of prefix-, suffix-, bifix-, and factor-free regular languages, Theoret. Comput. Sci. 449 (2012) 37-53.

[5] J. Brzozowski and Y. Ye, Syntactic complexity of ideal and closed languages, DLT 2011, eds. G. Mauri and A. Leporati LNCS 6795, (Springer Berlin / Heidelberg, 2011), pp. 117-128.

[6] O. Ganyushkin and V. Mazorchuk, Classical Finite Transformation Semigroups: An Introduction (Springer, 2009).

[7] M. Holzer and B. König, On deterministic finite automata and syntactic monoid size, Theoret. Comput. Sci. 327(3) (2004) 319-347.

[8] G. Jirásková and T. Masopust, On the state and computational complexity of the reverse of acyclic minimal DFAs, CIAA 2012, eds. N. Moreira and R. Reis LNCS 7381, (Springer, 2012), pp. 229-239. 
[9] G. Jirásková and T. Masopust, On the state complexity of the reverse of $\mathcal{R}$ - and $\mathcal{J}$-trivial regular languages, DCFS 2013, eds. H. Jurgensen and R. Reis LNCS 8031, (Springer, 2013), pp. 136-147.

[10] O. Klíma and L. Polák, On biautomata, Third Workshop on Non-Classical Models for Automata and Applications - NCMA 2011, Milan, Italy, July 18-July 19, 2011. Proceedings, eds. R. Freund, M. Holzer, C. Mereghetti, F. Otto and B. Palano 282, (Austrian Computer Society, 2011), pp. 153-164.

[11] B. Krawetz, J. Lawrence and J. Shallit, State complexity and the monoid of transformations of a finite set, http://arxiv.org/abs/math/0306416v1 (2003).

[12] A. N. Maslov, Estimates of the number of states of finite automata, Dokl. Akad. Nauk SSSR 194 (1970) 1266-1268 (Russian), English translation: Soviet Math. Dokl. 11 (1970), 1373-1375.

[13] J. Myhill, Finite automata and the representation of events., Wright Air Development Center Technical Report 57-624 (1957).

[14] J.-E. Pin, Syntactic semigroups, Handbook of Formal Languages, vol. 1: Word, Language, Grammar, eds. G. Rozenberg and A. Salomaa (Springer, 1997), pp. 679-746.

[15] J. Riordan, An introduction to combinatorial analysis (Wiley, 1958).

[16] T. Saito, $\mathcal{J}$-trivial subsemigroups of finite full transformation semigroups, Semigroup Forum 57 (1998) 60-68.

[17] M. Schützenberger, On finite monoids having only trivial subgroups, Inform. and Control 8 (1965) 190-194.

[18] I. Simon, Hierarchies of events with dot-depth one, PhD thesis, Dept. of Applied Analysis \& Computer Science, University of Waterloo (1972).

[19] I. Simon, Piecewise testable events, Proceedings of the 2nd GI Conference on Automata Theory and Formal Languages, (Springer-Verlag, London, UK, 1975), pp. $214-222$.

[20] S. Yu, Regular languages, Handbook of Formal Languages, vol. 1: Word, Language, Grammar, eds. G. Rozenberg and A. Salomaa (Springer, 1997), pp. 41-110. 